\title{
Collapse of ferromagnetism with Ti doping in $\mathrm{Sm}_{0.55} \mathrm{Sr}_{0.45} \mathrm{MnO}_{3}$ : A combined experimental and theoretical study
}

\author{
${ }^{1}$ Department of Physics, Serampore College, Serampore 712201, India \\ ${ }^{2}$ Saha Institute of Nuclear Physics, HBNI, \\ 1/AF Bidhannagar, Calcutta 700064, India.
}

(Dated: November 9, 2018)

We have investigated the effect of Ti doping on the transport properties coupled with the magnetic ones in $\mathrm{Sm}_{0.55} \mathrm{Sr}_{0.45} \mathrm{Mn}_{1-\eta} \mathrm{Ti}_{\eta} \mathrm{O}_{3}(0 \leq \eta \leq 0.04)$. The parent compound, $\mathrm{Sm}_{0.55} \mathrm{Sr}_{0.45} \mathrm{MnO}_{3}$, exhibits a first-order paramagnetic-insulator to ferromagnetic-metal transition just below $T_{\mathrm{c}}=128 \mathrm{~K}$. With substitution of Ti at Mn sites ( $B$-site), $T_{\mathrm{c}}$ decreases approximately linearly at the rate of $22 \mathrm{~K} \%^{-1}$ while the width of thermal hysteresis in magnetization and resistivity increases almost in an exponential fashion. The most spectacular effect has been observed for the composition $\eta=0.03$, where a magnetic field of only 1 $\mathrm{T}$ yields a huge magnetoresistance, $1.2 \times 10^{7} \%$ at $T_{c} \approx 63 \mathrm{~K}$. With increasing magnetic field, the transition shifts towards higher temperature, and the first-order nature of the transition gets weakened and eventually becomes crossover above a critical field $\left(H_{c r}\right)$ which increases with Ti doping. For Ti doping above 0.03, the system remains insulting without any ferromagnetic ordering down to $2 \mathrm{~K}$. The Monte-Carlo calculations based on a two-band double exchange model show that the decrease of $T_{\mathrm{c}}$ with Ti doping is associated with the increase of the lattice distortions around the doped Ti ions. 


\section{INTRODUCTION}

Perovskite manganites of the form $\mathrm{RE}_{1-x} \mathrm{AE}_{x} \mathrm{MnO}_{3}$ (RE: rare-earth ions and AE: alkalineearth ions) display rich varieties of physical phenomena owing to complex interplay between spin, charge, and orbital degrees of freedom. $\underline{\underline{1}} \underline{\underline{6}}$ The competition between these degrees of freedom is most prominently manifested in narrow-band system with large disorder. Usually, two types of disorders are considered in manganites. One is $A$-site disorder, namely the quenched disorder, that arises mainly due to the size mismatch between $\mathrm{RE}$ and $\mathrm{AE}$ cations and the other is $B(\mathrm{Mn})$-site disorder, originates due to the partial substitution of Mn by other transition metal ions with different spin and valence state. Though, $A$-site ions are not directly involve in charge conduction mechanism, several studies have shown that the disorder at $A$-site has a strong influence on different kinds of long-range ordering of manganese sublattice. Among these ordered phases, the charge-ordered (CO) state is most sensitive to $A$-site disorder while the ferromagnetic (FM)-metallic phase is rela-

tively weakly affected ${ }^{7-21}$. On the other hand, the doping at $B$-site induces local disorder directly into the Mn-O-Mn network and as a result, it has much stronger effect on magnetic, transport and other physical properties of the system as compared to $A$-site disorder. Only a few percent of $B$-site doping can bring about a drastic change in the electronic and magnetic properties without a significant change in the crystal structure. Several experiments have been performed on a large number of combination of reference states and $B$-site dopants $22-34$. On the basis of reference state, two classes of materials can be distinguished: (i) FM-metal at $x \approx 0.33-0.4$ and (ii) CO-insulator at around $x \approx 0.5^{\frac{35}{5}}$. In half-doped CO manganites, often substitution of small amount of $\mathrm{Cr} / \mathrm{Ni} / \mathrm{Ru}$ at Mn site dramatically suppresses the long-range CO state and drives the system into FM metallic state. On the contrary, the $B$-site doping in FM manganites may result in a strong suppression of ferromagnetism by localizing the charge carriers which lead to the formation of inhomogeneous and insulating magnetic ground state.

The effect of Mn-site doping on magnetic and transport properties has already been studied extensively but mostly on wideband FM manganites $\underline{28} \underline{34}$ However, the role of Mn-site doping in narrowband FM system, in particular, close to the multicritical point has not been studied in details. In the present work, we focus on a narrowband manganite, $\mathrm{Sm}_{0.55} \mathrm{Sr}_{0.45} \mathrm{MnO}_{3}$ (SSMO), which locates near the multicritical point where the three phases namely, FM-metal, CO-insulator and antiferromagnetic-insulator compete strongly with each other, to explore the role of Mn-site disorder on the FM phase $\underline{14}$-21 . The effect of $B$-site doping on magnetic and transport properties 
of $\mathrm{Sm}_{0.55} \mathrm{Sr}_{0.45} \mathrm{Mn}_{1-\eta} \mathrm{Ti}_{\eta} \mathrm{O}_{3}$ with $0 \leq \eta \leq 0.04$ has been studied systematically. The results show that FM-metal to paramagnetic (PM)-insulator transition in SSMO is first-order with transition temperature, $T_{c} \approx 128 \mathrm{~K}$. With the substitution of non-magnetic $\mathrm{Ti}^{4+}$, both the $\mathrm{FM}$ transition temperature, $T_{\mathrm{c}}$, and metal-insulator transition temperature (MIT), $T_{M I}$, decrease, while the thermal hysteresis width $(\Delta T)$ in electrical resistivity $(\rho)$ and magnetization $(M)$ increases drastically. Only $3 \%$ Ti doping increases $\Delta T$ from 4.5 to $23.4 \mathrm{~K}$. To the best of our knowledge, such a huge increase in $\Delta T$ due to the $B$-site substitution has not been reported earlier in any FM manganite. The application of external magnetic field $(H)$ shifts MIT towards higher temperature, leading to a field dependent phase boundary. Besides these experimental findings, the role of $B$-site doping on transport and magnetic properties has also been investigated using model Hamiltonian calculations. Our calculations based on Monte-Carlo technique using a two-band double exchange model including electron-phonon coupling, super-exchange interactions and quenched disorder reveal that with increasing $\mathrm{Ti}$ content, the lattice distortions around the Ti ions increases and as a result $T_{\mathrm{c}}$ decreases, which qualitatively agree with experimental results.

\section{EXPERIMENTS}

Polycrystalline $\mathrm{Sm}_{0.55} \mathrm{Sr}_{0.45} \mathrm{Mn}_{1-\eta} \mathrm{Ti}_{\eta} \mathrm{O}_{3}$ samples with $\eta=0-0.04$ were prepared by conventional solid-state reaction technique. The starting materials, $\mathrm{Sm}_{2} \mathrm{O}_{3}$ (pre-fired), $\mathrm{SrCO}_{3}, \mathrm{Mn}_{3} \mathrm{O}_{4}$ and $\mathrm{TiO}_{2}$ were mixed in a stoichiometric ratio and ground thoroughly in an agate mortar by using ethanol. The mixture was put in a platinum crucible and calcined in air at $1100^{\circ} \mathrm{C}$ for few days with intermediate grindings. The obtained powder was pulverized and sintered at $1200^{\circ} \mathrm{C}$ for $24 \mathrm{~h}$ to ensure the chemical homogeneity. Phase purity and the structural analysis of the samples were done by powder x-ray diffraction (XRD) technique with $\mathrm{Cu}-\mathrm{K}_{\alpha}$ radiation in a high resolution Rigaku X-ray diffractometer (TTRAX II). For all the studied compositions $(\eta=0-0.04)$, we did not observe any peak due to the impurity phase in the XRD pattern. The Rietveld refinement technique was used for structural analysis. The dc magnetization measurements were performed using a magnetic property measurement system (SQUID-VSM, Quantum Design). Resistivity measurements were performed by a conventional four-probe technique over a wide range of temperature for different applied magnetic fields up to $9 \mathrm{~T}$. We have measured transport and magnetic properties as functions of $H$ and $T$ for all the samples, but for clarity few of them are presented. 
TABLE I: Refined parameters for $\mathrm{Sm}_{0.55} \mathrm{Sr}_{0.45} \mathrm{Mn}_{1-\eta} \mathrm{Ti}_{\eta} \mathrm{O}_{3}(0.00 \leq \eta \leq 0.03)$ compound at room-temperature with Pnma space group. $a, b, c$ are the lattice parameters and $v$ is the unit cell volume. Numbers in the parenthesis are the statistical errors. $U_{i s o}$ is the isotropic atomic displacement parameter. $\chi^{2}$ is goodness of the fit.

\begin{tabular}{ccccc}
\hline \hline Composition & $\eta=0.00$ & $\eta=0.01$ & $\eta=0.02$ & $\eta=0.03$ \\
\hline$a(\AA)$ & $5.43027(11)$ & $5.43271(13)$ & $5.43433(9)$ & $5.43703(13)$ \\
$b(\AA)$ & $7.66747(14)$ & $7.66989(16)$ & $7.67356(11)$ & $7.67702(16)$ \\
$c(\AA)$ & $5.44146(9)$ & $5.44253(11)$ & $5.44352(9)$ & $5.44512(12)$ \\
$v\left(\AA^{3}\right)$ & $226.563(7)$ & $226.781(8)$ & $226.999(6)$ & $227.280(9)$ \\
$\chi^{2}(\%)$ & 3.34 & 3.25 & 3.17 & 3.17 \\
$U_{\text {iso }}\left(\AA^{2}\right), \mathrm{Sm}(\mathrm{Sr})$ & $0.0052(4)$ & $0.0067(4)$ & $0.0070(4)$ & $0.0077(4)$ \\
$U_{\text {iso }}\left(\AA^{2}\right), \mathrm{Mn}(\mathrm{Ti})$ & $0.0021(6)$ & $0.0049(6)$ & $0.0032(5)$ & $0.0048(6)$ \\
$U_{\text {iso }}\left(\AA^{2}\right), \mathrm{O} 1(\mathrm{O} 2)$ & $-0.003(2)$ & $0.007(2)$ & $0.002(2)$ & $0.0033(19)$ \\
\hline \hline
\end{tabular}

\section{RESULTS AND DISCUSSION}

\section{A. Crystal Structure}

Figure 1 shows the room-temperature powder x-ray diffraction of $\mathrm{Sm}_{0.55} \mathrm{Sr}_{0.45} \mathrm{Mn}_{1-\eta} \mathrm{Ti}_{\eta} \mathrm{O}_{3}$ for four compositions, $\eta=0,0.01,0.02$ and 0.03 as representatives. The diffraction patterns show that all the samples have a perovskite orthorhombic (space group Pnma) structure in which the atomic positions of $\mathrm{Sm}(\mathrm{Sr}): 4 c(x, 1 / 4, z), \mathrm{Mn}(\mathrm{Ti}): 4 b(0,0,1 / 2), \mathrm{O} 1: 4 c(x, 1 / 4, z)$ and $\mathrm{O} 2: 8 d(x, y, z)$ are used for indexing the Bragg peaks $\frac{36}{}$. The crystal structure of the samples does not change with Ti doping. However, with increasing Ti concentration, the lattice parameters $a, b, c$ and hence the unit cell volume increase as shown in the inset of Fig. 1. The increase of cell volume suggests the substitution of $\mathrm{Mn}^{4+}$ ions by $\mathrm{Ti}^{4+}$ ions, considering that the ionic radius of $\mathrm{Ti}^{4+}(0.605 \AA)$ is larger than that of the $\mathrm{Mn}^{4+}(0.530 \AA)$. The refined parameters are presented in the Table I for various Ti doping. $\mathrm{Ti}^{4+}$ ions partially and randomly substitute isovalent $\mathrm{Mn}^{4+}$ ions and it is believed that like other $\mathrm{Ti}$ doped manganites, the substitution of $\mathrm{Ti}$ in the present system also increases the average $(\mathrm{Mn}, \mathrm{Ti})-\mathrm{O}$ bond lengths, decreases $(\mathrm{Mn}, \mathrm{Ti})-\mathrm{O}-(\mathrm{Mn}, \mathrm{Ti})$ bond angle and hence reduces the bandwidth of the system ${ }^{30}$. 


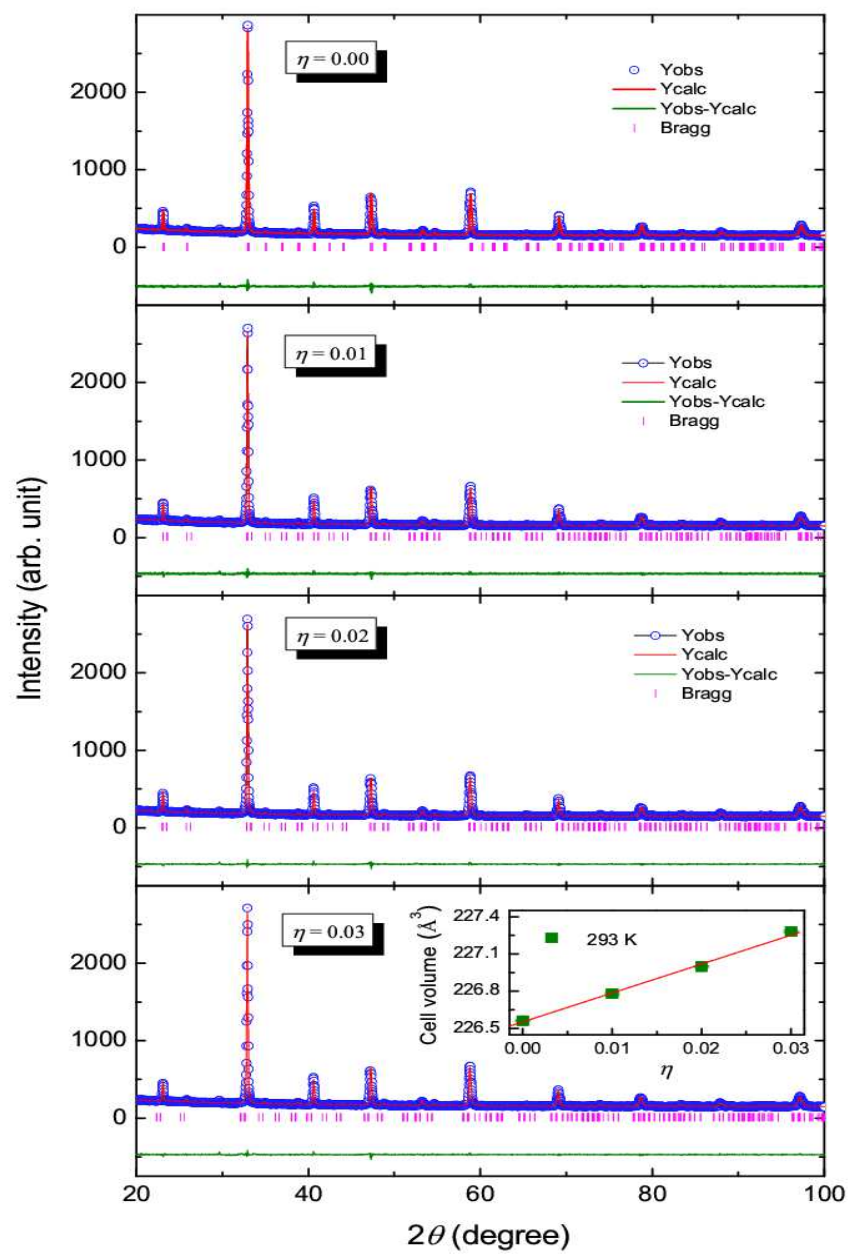

FIG. 1: (Color online) X-ray diffraction pattern of $\mathrm{Sm}_{0.55} \mathrm{Sr}_{0.45} \mathrm{Mn}_{1-\eta} \mathrm{Ti}_{\eta} \mathrm{O}_{3}(0.00 \leq \eta \leq 0.03)$ at room temperature. The bottom curves (Yobs-Ycalc) are due to difference between the observed data and the refinement data and the vertical bars indicate the Bragg peak positions for $\mathrm{Cu}-K_{\alpha_{1}}$ and $\mathrm{Cu}-K_{\alpha_{2}}$ radiations. Inset shows Ti doping dependence of the unit cell volume at room temperature.

\section{B. Magnetic and transport properties}

Figure 2(a) shows the temperature dependence of magnetization of $\mathrm{Sm}_{0.55} \mathrm{Sr}_{0.45} \mathrm{Mn}_{1-\eta} \mathrm{Ti}_{\eta} \mathrm{O}_{3}$ for $\eta=0.00,0.01,0.02$ and 0.03 . The parent compound, SSMO shows a sharp FM-PM transition at $T_{\mathrm{c}} \sim 128 \mathrm{~K}$, estimated as the temperature at which the temperature coefficient of magnetization $(\mathrm{d} M / \mathrm{d} T)$ exhibits a deep minimum [inset of Fig. 2(b)]. However, the magnetization data are not same in the warming and cooling cycles, but exhibit a strong irreversibility of $\sim 4.5 \mathrm{~K}$. The irreversibility in $M(T)$ curve demonstrates the first-order nature of FM transition in $\mathrm{Sm}_{0.55} \mathrm{Sr}_{0.45} \mathrm{MnO}_{3}$. 

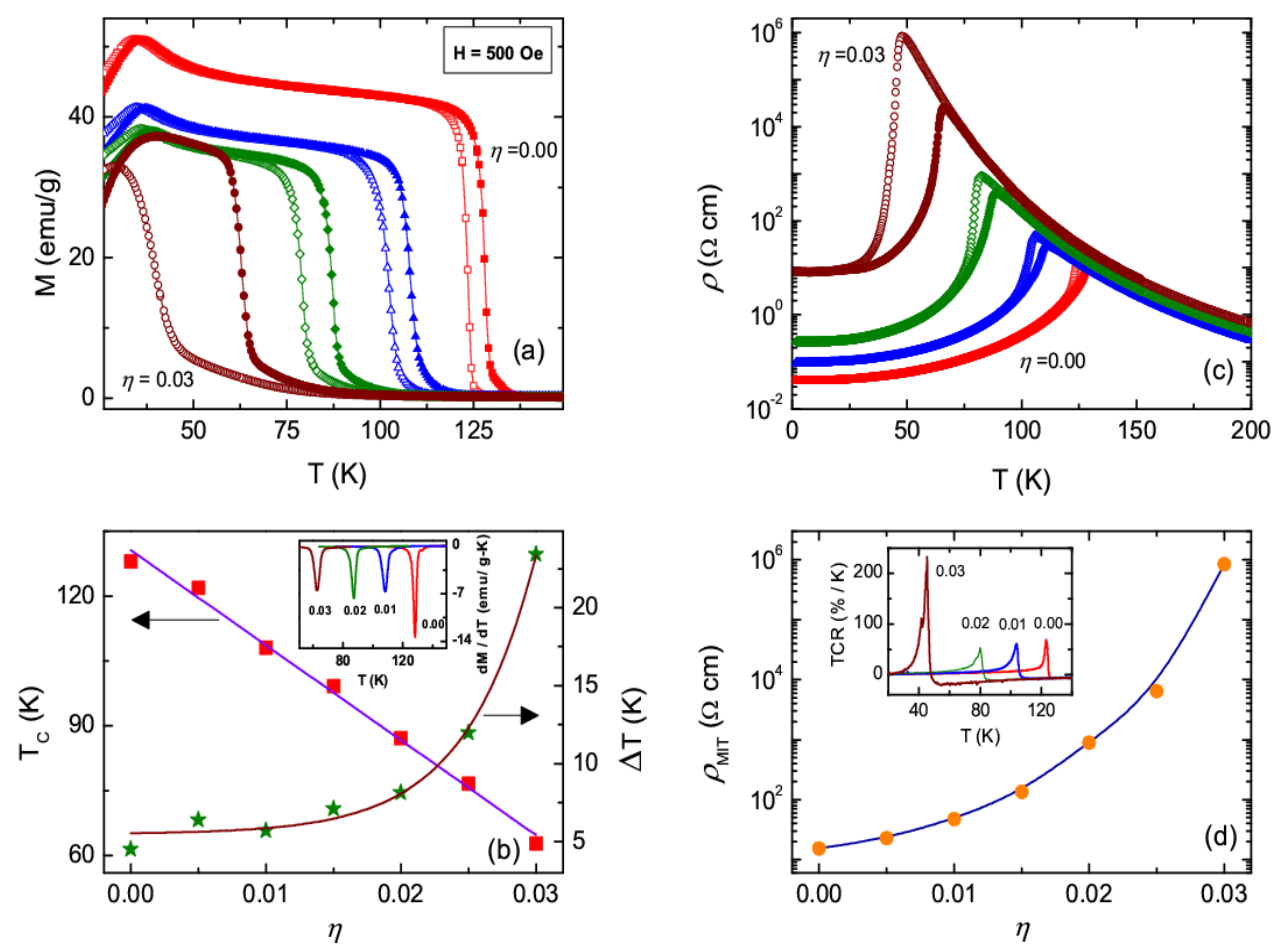

FIG. 2: (Color online) (a) Temperature $(T)$ dependence of magnetization $(M)$ of $\mathrm{Sm}_{0.55} \mathrm{Sr}_{0.45} \mathrm{Mn}_{1-\eta} \mathrm{Ti}_{\eta} \mathrm{O}_{3}$ for $\eta=0.0,0.01,0.02$ and 0.03. Closed and open symbols represent heating and cooling cycles, respectively. (b) Ferromagnetic-paramagnetic transition temperature, $T_{\mathrm{c}}$ (in the warming cycle) and thermal hysteresis width $(\Delta T)$ as a function of Ti concentration $(\eta)$. Inset shows $T$ dependence of $\mathrm{d} M / \mathrm{d} T$ for different $\eta$. (c) Temperature profile of resistivity $(\rho)$ for different $\eta$ both in warming (closed symbol) and cooling (open symbol) cycles. (d) Peak resistivity at metal insulator transition $\left(\rho_{M I T}\right)$ as a function of $\eta \cdot \rho_{M I T}$ is derived from the cooling cycle of $\rho(T)$ curve. Inset shows the temperature coefficient of resistivity (TCR) as a function of $T$ for different $\eta$.

With increasing $\eta$, the ferromagnetism is suppressed which is indicated through the reduction of magnetization as well as a strong decrease in $T_{\mathrm{c}}$. $T_{\mathrm{c}}$ is observed to decrease approximately linearly with $\eta$ at the rate of $22 \mathrm{~K}^{-1}$ [Fig. 2(b)], which is much higher than that observed in several other Ti doped FM manganites ${ }^{30}-32$. Not only the $T_{\mathrm{c}}$, the width of thermal hysteresis also changes drastically with Ti doping which is shown in Fig. 2(b). Remarkably, only 3\% Ti doping increases $\Delta T$ from $4.5 \mathrm{~K}$ to $23.4 \mathrm{~K}$. It may be mentioned that $M$ has also been measured for sample with slightly higher Ti concentration $(\eta=0.04)$ but no FM transition has been observed down to $2 \mathrm{~K}$. In the resistivity curve [Fig. 2 (c)], the MIT is observed at $T_{M I} \approx 129 \mathrm{~K}(\eta=0)$, corresponding 
to resistivity maximum. The presence of thermal hysteresis in $\rho(T)$ curve around $T_{M I}$ indicates that the MIT is first-order in nature. Similar to magnetization, as Ti substitution proceeds, $T_{M I}$ decreases linearly while the width of the thermal hysteresis in $\rho(T)$ increases exponentially. Depending on the degree of influence of $B$-site doping on charge conduction, the whole temperature region in $\rho(T)$ curve can be divided into three main parts. At low temperatures well below $T_{M I}, \rho$ increases sharply with increasing Ti content. As $\eta$ increases from 0 to 0.03 , the residual resistivity increases almost by a factor $10^{4}$. The value of residual resistivity $(\sim 8.5 \Omega \mathrm{cm})$ for $\eta=0.03$ is well above the Ioffe-Regel limit $\left(\sim 10^{-3} \Omega \mathrm{cm}\right)$ to observe metallic behavior, suggesting that the ground state is not a homogeneous ferromagnet rather it can be a coexistence of FM and short-range CO states. Similar to residual resistivity, the peak resistivity at MIT $\left(\rho_{M I T}\right)$ also enhances by a factor as high as $10^{5}$ with increasing $\eta$ from 0 to 0.03 , as shown in Fig. 2 (d). In the PM insulating state well above $T_{M I}$, the effect of Ti substitution on $\rho$ is relatively weaker as compared to that in the low-temperature region. For all the samples, the temperature coefficient of resistivity [TCR = $\left.\frac{1}{\rho}\left(\frac{d \rho}{d T}\right)\right]$ exhibits a very sharp peak at $T_{\mathrm{c}}$ [inset of Fig. 2(d)], expected for a first-order phase transition. From figure, one can see that the maximum value of TCR is almost same for $0 \leq \eta \leq 0.02$, but it abruptly increases for $\eta=0.03$. As MIT for $\eta=0.03$ is much sharper as compared to other compositions, TCR is very large for this composition in spite of large value of $\rho$. This behavior is quite unexpected. Normally, disordering in the active Mn-O-Mn network is supposed to broaden the FM transition.

The inset of Fig. 3 (a) shows magnetization hysteresis loop at $5 \mathrm{~K}$ for $\eta=0.00$ and 0.03 . We have measured $M(H)$ at $5 \mathrm{~K}$ for $\eta=0.00,0.01,0.02$ and 0.03 , but for clarity, only $\eta=0$ and 0.03 data are presented in the figure. As in the case of a typical soft ferromagnet, the magnetization of the samples with $\eta \leq 0.02$ increases rapidly with the application of field and tends to saturate at a relatively low field strength. However, for $\eta=0.03$, the nature of $M(H)$ curve at $5 \mathrm{~K}$ is not like a simple ferromagnet but it exhibits a metamagnetic transition along with field hysteresis. Ti substitution weakens the FM ordering of parent compound and may favor the formation of shortrange $\mathrm{CO}$ state. The saturation magnetization for different $\eta$ are determined by extrapolating the high field part of $M(H)$ curves to $H=0$. The estimated values of saturation magnetization are $3.51,3.46,3.45$ and $3.44 \mu_{B}$ per Mn atom for $\eta=0,0.01,0.02$ and 0.03 , respectively. These values are slightly lower than their respective theoretical values of $3.55,3.52,3.49$ and $3.46 \mu_{B}$ per Mn atom, suggesting that the decrease of magnetization with Ti doping is not only due the dilution of $\mathrm{Mn}^{4+}$ atom but also due to the weakening of exchange coupling. In the vicinity of $T_{\mathrm{c}}$, $M(H)$ isotherms for $\eta=0$ and 0.02 are presented in Figs. 3 (a) and (b), respectively. $M(H)$ curves 

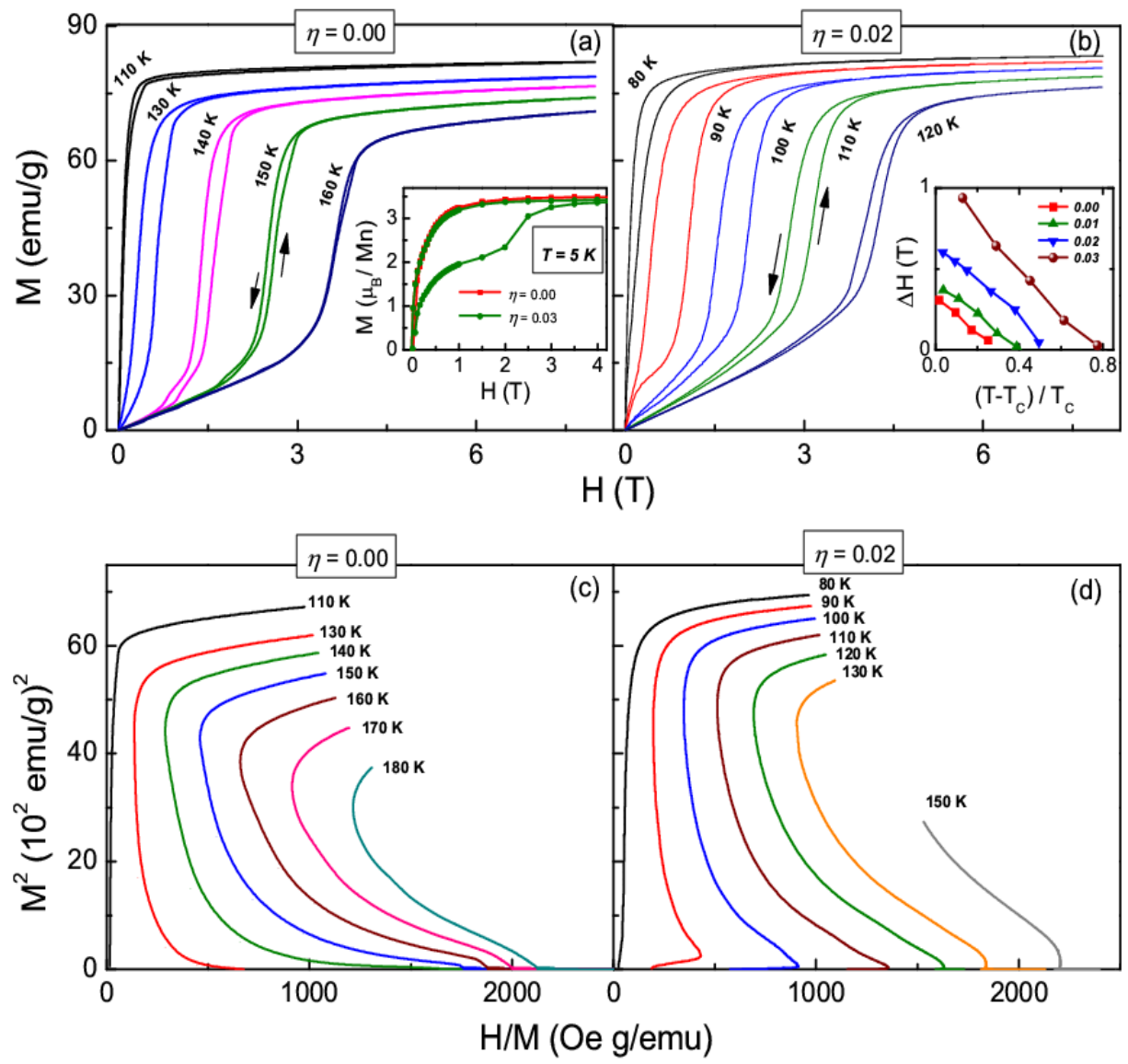

FIG. 3: (Color online) $M(H)$ isotherms of $\mathrm{Sm}_{0.55} \mathrm{Sr}_{0.45} \mathrm{Mn}_{1-\eta} \mathrm{Ti}_{\eta} \mathrm{O}_{3}$ for (a) $\eta=0.00$ and (b) $\eta=$ 0.02. Inset of figure (a) represents $M(H)$ hysteresis loop measured at $5 \mathrm{~K}$, while the inset of figure (b) shows hysteresis width $(\Delta H)$ of $M(H)$ isotherms between increasing and decreasing fields as a function of reduced temperature, $\left(T-T_{c}\right) / T_{c}$. Arrott plots $\left(M^{2}\right.$ vs. $\left.H / M\right)$ for $(\mathrm{c}) \eta=$ 0.00 and $(\mathrm{d}) 0.02$.

below $T_{\mathrm{c}}$ are typical of a ferromagnet with small hysteresis between increasing and decreasing field. Initially, $M$ increases rapidly with $H$ and then tends to saturate and the saturation value of $M$ gradually decreases with increasing temperature. Above $T_{\mathrm{c}}$, we observe S-shaped $M(H)$ isotherms, which indicates a metamagnetic phase transition. With increasing $H$, first $M$ increases almost linearly and then suffers a step-like jump, indicative of reentrant ferromagnetism. Such a step-like jump in $M$ along with the hysteresis are the manifestation of field-induced first-order PM-FM phase transition. The inset of Fig. 3 (b) shows the temperature [reduced temperature, $\left.\left(T-T_{c}\right) / T_{c}\right]$ dependence of width of the field hysteresis $(\Delta H)$ in $M(H)$ isotherms for different $\eta$. For $\eta=0, \Delta H$ just above $T_{\mathrm{c}}(\sim 128 \mathrm{~K})$ is $\sim 0.3 \mathrm{~T}$, which decreases almost linearly with increasing 
$T$ and eventually vanishes at a temperature that is around $1.3 T_{\mathrm{c}} . \Delta H$ is observed to increase with increasing $\eta$ but decreases with increase in $T$ almost at the same rate as that for $\eta=0$. Figures 3 (c) and (d) show the Arrott plots $\left(M^{2}\right.$ vs. $\left.H / M\right)$, which offers a criterion for determining whether FM to PM phase transition is first-order or second-order purely by magnetic method 37 . According to Banerjee criterion, if the slope of the Arrott plot is positive then the FM transition is second-order in nature and for a first-order transition the slope is negative ${ }^{38}$. The undoped compound shows a negative slope in $M^{2}$ vs. $H / M$ plot and this behavior persists for $\eta \leq 0.03$, which means that FM transition in all samples is first-order.
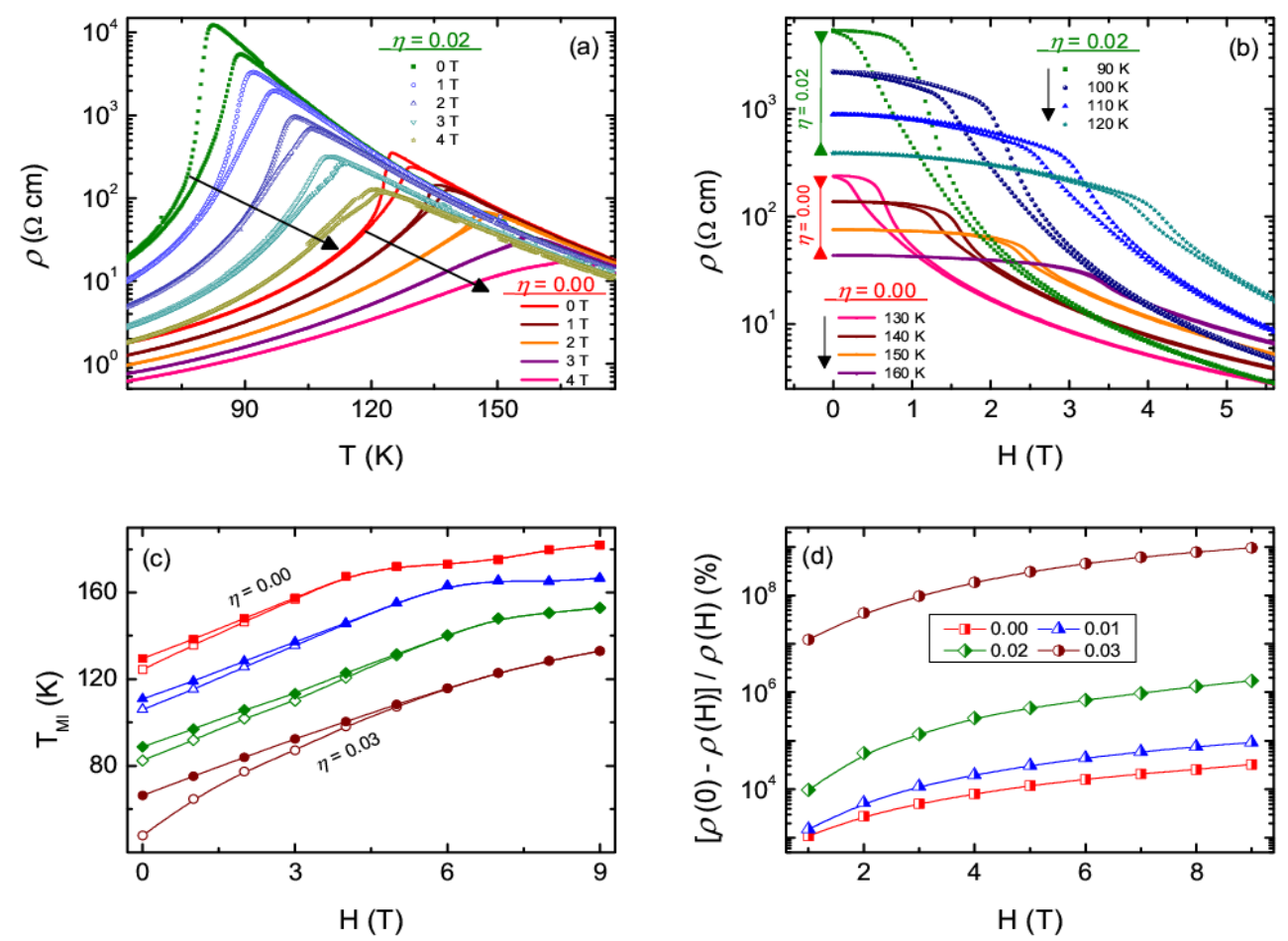

FIG. 4: (Color online) (a) $\rho$ vs $T$ curves at different $H(=0,1,2,3$ and $4 \mathrm{~T}$ ) for $\eta=0.00$ (lines) and 0.02 (symbols). Data measured during warming and cooling cycles are shown in the figure.

The arrow indicates the direction of increasing field. (b) $H$ dependence of $\rho$ at different temperatures for $\eta=0.00$ (lines) and 0.02 (symbols). (c) The variation of metal-insulator transition temperature $\left(T_{M I}\right)$ with $H$ for different $\eta$. Closed and open symbols are the $T_{M I}$ 's derived from the heating and cooling cycles of $\rho(T)$ curves, respectively. (d) Magnetoresistance $[(\rho(0)-\rho(H)) / \rho(H)]$ as a function of $H$ for different $\eta$. It is calculated at $T=T_{M I}(H=0)$.

We now investigate the effect of external magnetic field on FM phase transition in $\mathrm{Sm}_{0.55} \mathrm{Sr}_{0.45} \mathrm{Mn}_{1-\eta} \mathrm{Ti}_{\eta} \mathrm{O}_{3}$ $(0 \leq \eta \leq 0.03)$. The temperature and field dependence of $\rho$ for different $\eta(0$ and 0.02$)$ are shown in 
Figs. 4 (a) and (b). Figure 4 (a) shows that the effect of $H$ on $\rho$ is maximum in the vicinity of $T_{M I}$, whereas $\rho$ remains almost unchanged well above $T_{M I}$. For all samples, the peak resistivity and resistivity below $T_{M I}$ are observed to reduce strongly with field. Well below $T_{M I}$, application of magnetic field enhances the spin-polarized tunneling through grain boundaries and as a result, residual resistivity decreases rapidly with field. As shown in Fig. 4 (b), resistivity evolves with $H$ in an opposite way as that of magnetization [Figs. 3 (a) and (b)]. Just above $T_{\mathrm{c}}, \rho$ drops sharply with $H$ along with field hysteresis, a consequence of first-order phase transition. With increasing temperature, the sharpness of the field-induced change in $\rho$ diminishes, the width of the field hysteresis gradually becomes narrow and finally vanishes above a critical temperature, $T_{c r}$. The resultant $T_{M I}-H$ phase diagram for various Ti concentrations is plotted in Fig. 4 (c). As $H$ increases, the width of thermal hysteresis in $\rho$ gradually decreases and the two phase transition lines, corresponding to the warming and cooling processes, merge to one another at a critical magnetic field $\left(H_{c r}\right)$. This feature indicates that external field suppresses the first-order nature of the transition and the transition becomes a crossover above $H_{c r}$, and the value of critical field $H_{c r}$ increases from 4 to $6 \mathrm{~T}$ as $\eta$ increases from o to 0.03. In the regime of $H<H_{c r}, T_{M I}$ for all samples increases linearly with $H$ at an average rate of $9 \mathrm{~K} / \mathrm{T}$ but at a slower rate above $H_{c r}$. We have also calculated magnetoresistance $(\mathrm{MR})$ at $T=T_{M I}(H=0)$ for $0 \leq \eta \leq 0.03$. Here, MR is defined as $M R=\Delta \rho / \rho(H)=[\rho(0)-\rho(H)] / \rho(H) \times 100 \%$, where $\rho(0)$ and $\rho(H)$ are the values of resistivity at zero field and at an applied field $H$, respectively. Figure 4 (d) shows the typical magnetic field dependence of MR for different $\eta$. For $\eta=0$, the value of MR at $H=1 \mathrm{~T}$ is $1.1 \times 10^{4}$ $\%$, which increases with $H$ and becomes $3.2 \times 10^{4} \%$ for $H=9$ T. MR enhances with Ti doping and the most fascinating effect is observed for the composition $\eta=0.03$, where MR reaches to $1.2 \times 10^{7}$ $\%$ for $H=1 \mathrm{~T}$ only and it becomes $\sim 10^{9} \%$ for $H=9 \mathrm{~T}$. The observed value of MR is much higher as compared with several other FM manganites.

\section{Theoretical Simulation}

We consider a two-band model Hamiltonian in two dimensions for manganites in the large Hund's coupling limit $\left(J_{H} \rightarrow \infty\right)^{2, \underline{42}}$ to study the role of Ti doping on transport and magnetic properties of SSMO:

$$
\begin{aligned}
\mathcal{H}=- & \sum_{\langle i j\rangle \sigma}^{\alpha \beta} t_{\alpha \beta}^{i j} d_{i \alpha \sigma}^{\dagger} d_{j \beta \sigma}+J \sum_{\langle i j\rangle} \mathbf{S}_{i} \cdot \mathbf{S}_{j} \\
& -\lambda \sum_{i} \mathbf{Q}_{i} \cdot \boldsymbol{\tau}_{i}+\frac{K}{2} \sum_{i} \mathbf{Q}_{i}^{2}+\sum_{i} \epsilon_{i} n_{i},
\end{aligned}
$$


where $e_{g}$ electrons hop between nearest neighbor sites $i$ and $j$ with amplitude $t_{\alpha \beta}^{i j}$ (for two orbitals $a$ and $b$ ). The hopping amplitudes $t_{\alpha \beta}^{i j}$ depend upon the orientation of Mn $t_{2 g}$ spins at the sites $i$ and $j$. For details please see Ref. 22. $J$ and $\lambda$ are anti-ferromagnetic super-exchange interactions between $\mathrm{Mn} \mathrm{t}_{2 g}$ spins $\left(\mathbf{S}_{\mathrm{i}}\right)$ and electron-phonon interactions between the $e_{g}$ electrons and the JahnTeller phonons $\mathbf{Q}_{i}$ in the adiabatic limit, respectively. We treat all $\mathbf{S}_{\mathbf{i}}$ (with $\left|\mathbf{S}_{i}\right|=1$ ) and $\mathbf{Q}_{i}$ (with stiffness of the Jahn Teller modes $K=1)$ as classical $\underline{39} \underline{40}$, and measure all parameters $(J, \lambda$, and temperature $T$ ) in the units hopping amplitude $t_{\text {aa. }}$.
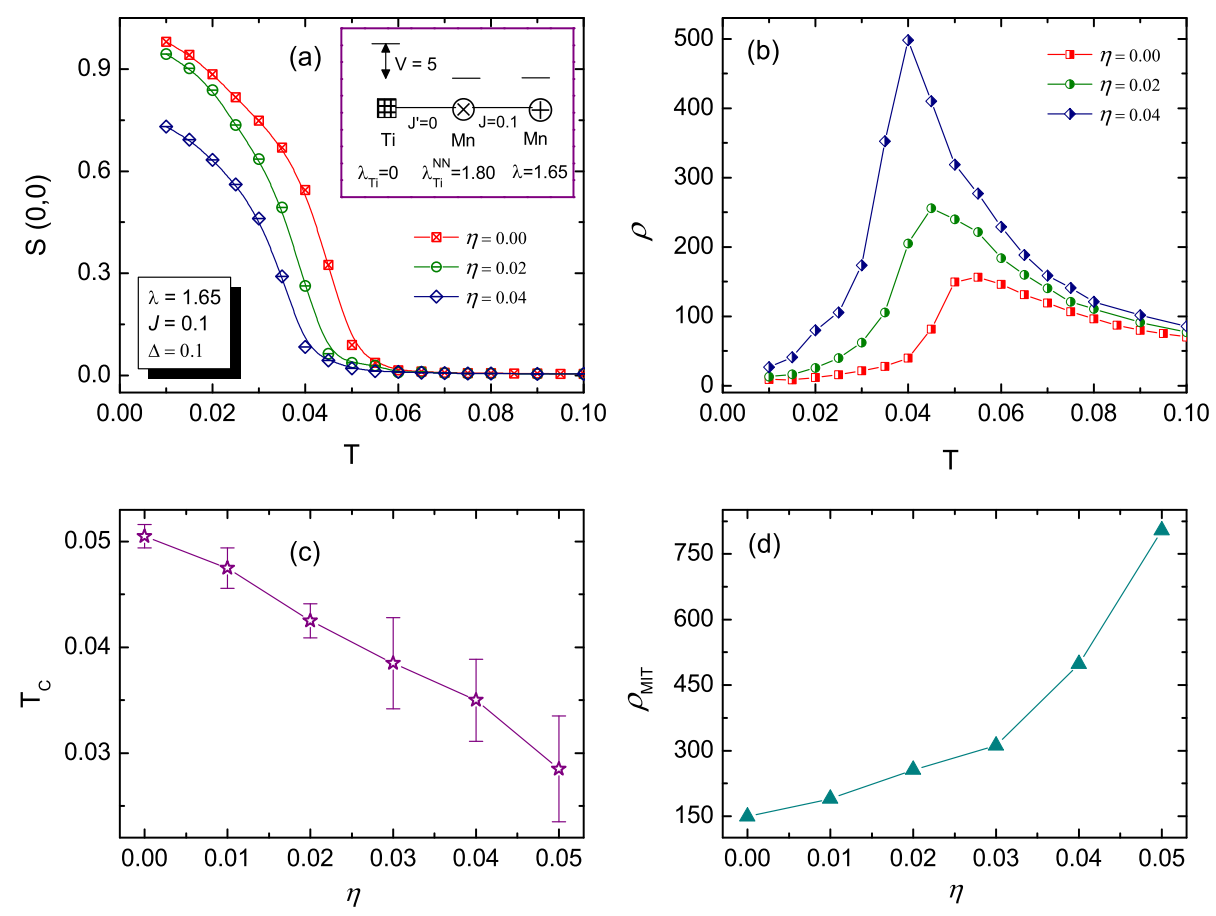

FIG. 5: (Color online) Temperature dependence of (a) ferromagnetic structure factor $[S(0,0)]$ and (b) resistivity $(\rho)$ for dopant concentration $\eta=0.00,0.02$, and 0.04. (c) Ferromagnetic transition temperature, $T_{\mathrm{c}}$, with various dopant concentrations $\eta$. (d) Peak resistivity at metal insulator transition $\left(\rho_{\mathrm{MIT}}\right)$ as a function of $\eta$. Schematic figure in the inset of (a) shows the relevant levels on Ti and Mn sites, and the coupling between these atoms. For notations please see the text.

This minimal model Hamiltonian $\mathcal{H}$ reproduces the correct sequence of magnetic phases $35,41,43$. Typical $\lambda \sim 1.6-1.7$ values with $J=0.1$ reproduce the colossal magnetoresistive properties of intermediate bandwidth manganites qualitatively $\underline{\underline{43}}$. For $\lambda=1.65$ and $J=0.1$, CE-type insulating phase can be reproduced for electron density $n=0.5$, whereas the FM window spans over in the range $0.6<n<0.7$ similar to intermediate bandwidth manganites. Here, we will concentrate our 
calculations for $n=0.65$ at which FM $T_{\mathrm{c}}$ is maximum. Recall that in ( $\left.\mathrm{Sm}, \mathrm{Sr}\right)$ manganite system, $T_{\mathrm{c}}$ is optimum for $n=0.55 \underline{\underline{16}}$ and our experiments are carried out at that electron density. The effect of A-site disorder (due to mismatch between the ionic radii of $\mathrm{Sm}^{3+}$ and $\mathrm{Sr}^{2+}$ ) is taken into account by adding $\sum_{i} \epsilon_{i} n_{i}$ in the Hamiltonian where $\epsilon_{i}$ is the quenched binary disorder potential with values $\pm \Delta$. We use $\Delta=0.1$ and also checked our calculations for $\Delta=0$ and 0.2 to show that strong quenched disorder suppresses $T_{\mathrm{c}}$ more rapidly with Ti doping.

Next, in order to incorporate the effect of non-magnetic B-site dopants $\left(\mathrm{Ti}^{4+}\right.$ in the present case), we modify the Hamiltonian as shown schematically in the inset of Fig. 5 (a). A large energy level $V(=5)$ is used at Ti sites, by adding $V \sum_{i} n_{i}$ to the Hamiltonian $\stackrel{42}{ }$. For $V=5$, the electron density at the impurity site is close to zero. The electron-phonon coupling is irrelevant at Ti sites, and for this reason we use $\lambda$ at impurity sites $\lambda_{\mathrm{Ti}}=0$. Super-exchange interaction $\left(J^{\prime}\right)$ between

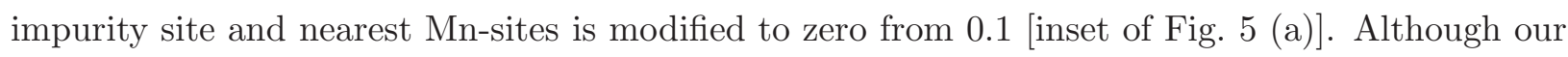
Hamiltonian has spin moment at each impurity site, but that moment is very weakly connected to rest of the system due to $J^{\prime}=0$ and large on site potential $V(=5)$. So, these moments at impurity sites do not affect the magnetism and are not taken into account while calculating the ferromagnetic order. From our experimental results, it is clear that with Ti doping, the unit cell volume of the system increases (inset of Fig. 10 and as a result, the bandwidth of the system decreases 30 . In order to take take this effect into account, we modify the $\lambda$ values at the $\mathrm{Mn}$ sites $\left(\lambda_{\mathrm{Ti}}^{\mathrm{NN}}=1.80\right)$ those are nearest neighbor to Ti ions. Recall that $\lambda$ is measured in units of kinetic energy, and thus larger $\lambda$ corresponds to smaller bandwidth. Large $\lambda_{\mathrm{Ti}}^{\mathrm{NN}}$ helps in localizing the electrons at those sites and as a results $\mathrm{Mn}^{3+}$ look-a-like ions surrounds the $\mathrm{Ti}^{4+}$ ions that minimizes the Coulomb repulsion.

We use an exact diagonalization scheme to the itinerant electron system for each configuration of the background classical variables $\mathbf{S}_{\mathrm{i}}$ and $\mathbf{Q}_{\mathbf{i}}$. We use a Monte Carlo sampling technique based on the traveling cluster approximation $\underline{43} \underline{44}$ to access large system sizes. All physical quantities like ferromagnetic structure factor and resistivity are thermally averaged over ten different samples (starting from ten different initial realizations of the quenched disorder and classical variables).

The temperature dependence of the ferromagnetic structure factor $S(0,0)$ and resistivity $\rho$ with Ti concentration $\eta$ is shown in Figs. 5 (a) and (b) respectively. $S(0,0)$ is obtained by calculating $S(\mathbf{q})=\frac{1}{(N-\eta)^{2}} \sum_{i j} \mathbf{S}_{\mathbf{i}} \cdot \mathbf{S}_{\mathbf{j}} \mathrm{e}^{i \mathbf{q} \cdot\left(\mathbf{r}_{\mathbf{i}}-\mathbf{r}_{\mathbf{j}}\right)}$ at wave vector $\mathbf{q}=(0,0)$ for Mn sites. The resistivity, in units of $\hbar a / \pi e^{2}$ ( $a$ : lattice constant), is obtained from the $d c$ limit of the conductivity (calculated using the Kubo-Greenwood formalism) $\underline{45} \underline{\underline{46}}$. The $T_{\mathrm{c}}$ decreases approximately linearly and the resistivity peak increases very fast with $\eta$ as shown in Figs. 5 (c) and (d), respectively and agrees qualitatively 
with our experiments.
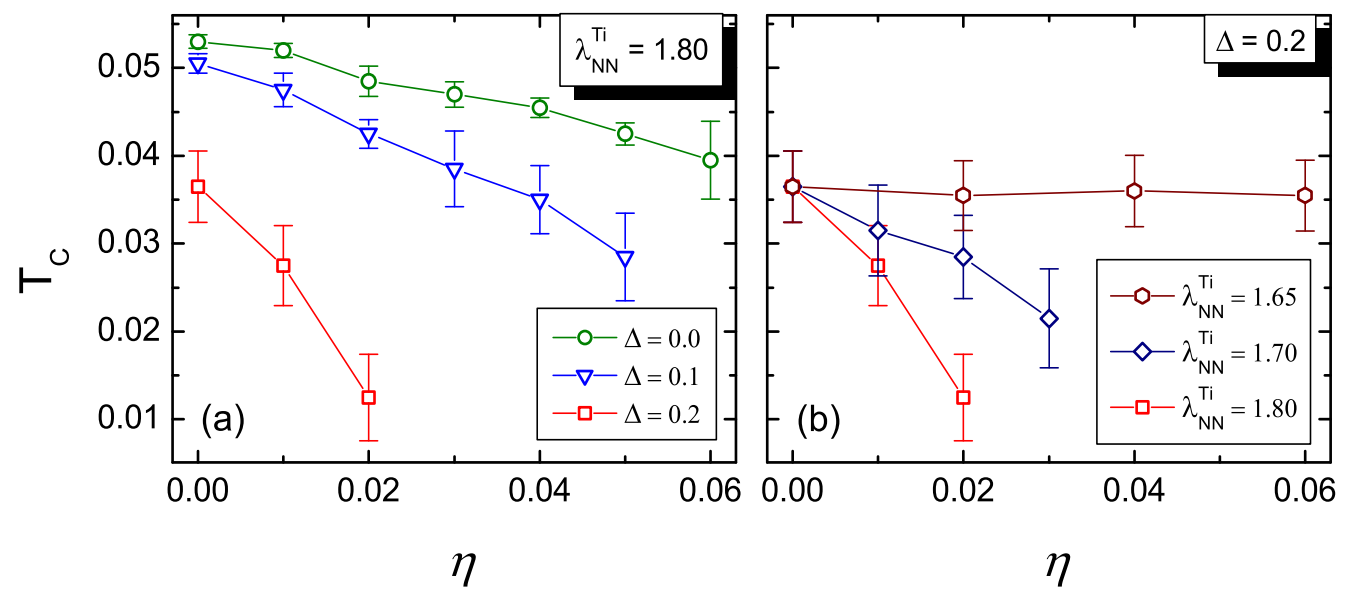

FIG. 6: (Color online) Variation of $T_{\mathrm{c}}$ with $\eta$ for $\lambda=1.65$ and $J=0.1$ : (a) different $\Delta$ values

$$
\text { (using } \lambda_{\mathrm{Ti}}^{\mathrm{NN}}=1.80 \text { ) and (b) different } \lambda_{\mathrm{Ti}}^{\mathrm{NN}} \text { values (using } \Delta=0.2 \text { ) }
$$

For $\Delta=0.2(\Delta=0)$ and $\lambda_{\mathrm{Ti}}^{\mathrm{NN}}=1.80$, the FM $T_{\mathrm{c}}$ decreases faster (slower) than $\Delta=0.1$ case as shown in Fig. 6 (a). This shows that disorder also plays an important role in decreasing the FM $T_{\mathrm{c}}$. We also use $\lambda_{\mathrm{Ti}}^{\mathrm{NN}}=1.70$ for $\Delta=0.2$ case and find that the FM $T_{\mathrm{c}}$ decreases linearly, albeit up to $\eta=0.03$, as shown in Fig. 6 (b). But for $\lambda_{\mathrm{Ti}}^{\mathrm{NN}}=1.65$ (i.e., without modifying $\lambda$ at nearest neighbour Mn ions of Ti site) the FM $T_{\mathrm{c}}$ remains more or less same until $\eta=0.06$ for $\Delta=0.2$. So, we believe that lattice distortions around the Ti ions increase, which localize the electrons and as a result the FM $T_{\mathrm{c}}$ decreases with Ti doping.

\section{CONCLUSION}

The effect of Ti doping on the magnetotransport properties of $\mathrm{Sm}_{0.55} \mathrm{Sr}_{0.45} \mathrm{Mn}_{1-\eta} \mathrm{Ti}_{\eta} \mathrm{O}_{3}(0 \leq$ $\eta \leq 0.03)$ has been studied. All these samples undergo first-order FM-metal to PM-insulator transition at $T_{\mathrm{c}}\left(\right.$ or $T_{M I}$ ) along with hysteresis. With increasing Ti concentration, $T_{\mathrm{c}}$ decreases linearly while the magnetoresistance increases very rapidly. Our theoretical calculations show that the FM $T_{\mathrm{c}}$ decreases due to the increase of lattice distortion around the Ti ions. The application of external field $H$ stabilizes the FM phase and thus weakens the first-order nature of the transition. The critical magnetic field where the first-order transition becomes a crossover increases with $\mathrm{Ti}$ doping. 
* psphysics1981@gmail.com

$\dagger$ nazirkhan91@gmail.com

$\ddagger$ kalpataru.pradhan@saha.ac.in

$\S$ prabhat.mandal@saha.ac.in

1 C.N.R. Rao, B. Raveau, Colossal Magnetoresistance, Charge Ordering and Related Properties of Manganese Oxides, (World Scientific, Singapore, 1998).

2 E. Dagotto, T. Hotta, and A. Moreo, Phys. Rep. 344, 1 (2001).

3 M. B. Salamon and M. Jaime, Rev. Mod. Phys. 73, 583 (2001).

4 E. Dagotto, Science 309, 257 (2005).

5 Y. Tokura, Rep. Prog. Phys. 69, 797 (2006).

6 P. K. Siwach, H. K. Singh, and O. N. Srivastava, J. Phys.: Condens. Matter 20, 273201 (2008).

7 H. Y. Hwang, S. W. Cheong, P. G. Radaelli, M. Marezio, and B. Batlogg, Phys. Rev. Lett. 75, 914 (1995).

8 Y. Tokura, H. Kuwahara, Y. Moritomo, Y. Tomioka, and A. Asamitsu, Phys. Rev. Lett. 763184 (1996).

9 L. M. Rodriguez-Martinez and J. P. Attfield, Phys. Rev. B 54, R15622 (1996); 58, 2426 (1998); 63, $024424(2000)$.

10 J. P. Attfield, Int. J. Inorg. Mater. 3, 1147 (2001).

11 Y. Tomioka, Y. Okimoto, J. H. Jung, R. Kumai, and Y. Tokura, Phys. Rev. B 68, 094417 (2003).

12 D. Akahoshi, M. Uchida, Y. Tomioka, T. Arima, Y. Matsui, and Y. Tokura, Phys. Rev. Lett. 90, 177203 (2003).

13 G. L. Liu, J. S. Zhou, and J. B. Goodenough, Phys. Rev. B 70, 224421 (2004).

14 Y. Tomioka and Y. Tokura, Phys. Rev. B 70, 14432 (2004).

15 L. M. Fisher, A. V. Kalinov, I. F. Voloshin, N. A. Babushkina, D. I. Khomshkii, Y. Zhang, and T. T. M. Palstra, Phys. Rev. B 70, 212411 (2004).

16 Y. Tomioka, H. Hiraka, Y. Endoh, and Y. Tokura, Phys. Rev. B 74, 104420 (2006).

17 P. Sarkar, P. Mandal, A. K. Bera, S. M. Yusuf, L. S. S. Chandra, and V. Ganesan, Phys. Rev. B 78, 012415 (2008).

18 L. Demkó, I. Kézsmárki, G. Mihály, N. Takeshita, Y. Tomioka, and Y. Tokura, Phys. Rev. Lett. 101, 037206 (2008).

19 P. Sarkar, P. Mandal, A. K. Bera, S. M. Yusuf, S. Arumugam, C. Q. Jin, T. Ishida, and S. Noguchi, Phys. Rev. B 79, 144431 (2009).

20 P. Sarkar, S. Arumugam, P. Mandal, A. Murugeswari, R. Thiyagarajan, S. E. Muthu, D. M. Radheep, C. Ganguli, K. Matsubayshi, and Y. Uwatoko, Phys. Rev. Lett. 103, 057205 (2009).

21 N. Khan, P. Sarkar, A. Midya, P. Mandal, and P. K. Mohanty, Sci. Rep. 7, 45004 (2017).

22 Y. Moritomo, A. Machida, S. Mori, N. Yamamoto, and A. Nakamura, Phys. Rev. B 60, 9220 (1999). 
23 T. Kimura, R. Kumai, Y. Okimoto, Y. Tomioka, and Y. Tokura Phys. Rev. B 62, 15021 (2000).

24 A. Machida, Y. Moritomo, K. Ohoyama, T. Katsufuji, and A. Nakamura, Phys. Rev. B 65, 064435 (2002).

25 H. Sakai, K. Ito, R. Kumai, and Y. Tokura, Phys. Rev. B 76, 155112 (2007).

26 C. L. Lu, X. Chen, S. Dong, K. F. Wang, H. L. Cai, J.-M. Liu, D. Li, and Z. D. Zhang Phys. Rev. B 79, 245105 (2009).

27 C. Lu , N. Hu , M. Yang , S. Xia , H. Wang, J. Wang , Z. Xia , and J. M. Liub , Sci. Rep. 4, 4902 (2014).

28 A. Maignan, F. Damay, A. Barnabe, C. Martin, M, Hervieu, and B. Raveau, Phil. Trans. R. Soc. Lond. A 356, 1635 (1998), and references herein.

29 C. Martin, A. Maignan, M. Hervieu, C. Autret, B. Raveau, and D. I. Khomskii, Phys. Rev. B 63, 174402 (2001).

30 M. S. Kim, J. B. Yang, Q. Cai, X. D. Zhou, W. J. James, W. B. Yelon, P. E. Parris, D. Buddhikot, and S. K. Malik, Phys. Rev. B 71, 014433 (2005).

31 D. N. H. Nam, L. V. Bau, N. V. Khiem, N. V. Dai, L. V. Hong, N. X. Phuc, R. S. Newrock, and P. Nordblad, Phys. Rev. B 73, 184430 (2006).

32 D. N. H. Nam, N. V. Dai, T. D. Thanh, L. T. C. Tuong, L. V. Hong, N. X. Phuc, H. S. Hong, and N. V. Khien, Phys. Rev. B 77, 224420 (2008).

33 M. M. Saber, M. Egilmez, A. I. Mansour, I. Fan, K. H. Chow, and J. Jung, Phys. Rev. B 82, 172401 (2010).

34 I. Dhiman , A. Das, A. K. Nigam, and R. K. Kremer, J. Magn. Magn. Mater. 334, 21 (2013).

35 K. Pradhan, A. Mukherjee, and P. Majumdar, Europhys. Lett. 84, 37007 (2008).

36 G. Murugesan, R. Nithya, and S. Kalainathan, Powder Diffraction 31, 77 (2016).

37 A. Arrott and J. E. Noakes, Phys. Rev. Lett. 19, 786 (1967).

38 B. K. Banerjee, Phys. Lett. 12, 16 (1964).

39 S. Yunoki, J. Hu, A. L. Malvezzi, A. Moreo, N. Furusaki, and E. Dagotto, Phys. Rev. Lett. 80, 845 (1998).

40 E. Dagotto, S. Yunoki, A. L. Malvezzi, A. Moreo, J. Hu, S. Capponi, D. Poilblanc, and N. Furukawa, Phys. Rev. B 58, 6414 (1998).

41 S. Yunoki, T. Hotta, and E. Dagotto, Phys. Rev. Lett. 84, 3714 (2000).

42 K. Pradhan, A. Mukherjee, and P. Majumdar, Phys. Rev. Lett. 99, 147206 (2007).

43 K. Pradhan and S. Yunoki, Phys. Rev. B 96, 214416 (2017).

44 S. Kumar and P. Majumdar, Eur. Phys. J. B 50, 571 (2006).

45 G. D. Mahan, Quantum Many Particle Physics (Plenum Press, New York, 1990).

46 S. Kumar and P. Majumdar, Europhys. Lett. 65, 75 (2004). 